\title{
Uso de Resíduo Industrial a Base de Resina Fenólica como Carga para o Polipropileno
}

\author{
André P. Cavalcante \\ Instituto de Macromoléculas, UFRJ \\ Leonardo B. Canto \\ Departamento de Engenharia de Materiais, UFSCar
}

\begin{abstract}
Resumo: O objetivo deste estudo foi analisar a viabilidade técnica do uso de resíduo industrial composto de resina fenólica, lignocelulose, carbonato de cálcio, talco, mica e negro de fumo como carga para o polipropileno (PP). Compósitos de PP com 10,15 e $20 \%$ em massa do resíduo foram processados em extrusora de rosca dupla e moldados por injeção. Os compósitos moldados foram caracterizados por microscopia eletrônica de varredura (MEV), análises térmicas (DSC e TGA), ensaios mecânicos (tração, flexão e impacto) e ensaio termo-mecânico (HDT). Os compósitos moldados apresentaram partículas do resíduo com tamanhos inferiores a 500 um dispersas na matriz de polipropileno. As partículas do resíduo atuaram como agentes de nucleação da cristalização do PP. Os compósitos apresentaram maior resistência à degradação térmica, balanço de rigidez e resistência com aumento do módulo sob flexão e diminuição da resistência à tração, manutenção da resistência ao impacto sob entalhe, e aumento da temperatura de distorção ao calor (HDT). O desempenho dos compósitos demonstrou que o resíduo industrial de resina fenólica pode ser empregado como carga funcional para o PP. Além disso, os compósitos desenvolvidos representam uma alternativa para a reciclagem do resíduo industrial de resina fenólica.
\end{abstract}

Palavras-chave: Compósitos de polipropileno, resina fenólica, reciclagem.

\section{Use of Industrial Waste Based on Phenolic Resin as Filler for Polypropylene}

Abstract: The aim of this study was to assess the technical feasibility of using industrial waste composed of phenolic resin, lignocellulose, calcium carbonate, talc, mica and carbon black as filler for polypropylene (PP). Composites of PP with 10, 15 and 20 wt\% of the waste were processed in a twin-screw extruder followed by injection molding. Molded composites were characterized by scanning electron microscopy (SEM), thermal analyses (DSC and TGA), mechanical tests (tensile, flexural and impact) and thermo-mechanical test (HDT). The composites showed residue particles with sizes less than $500 \mu \mathrm{m}$ dispersed in the PP matrix. The residue particles acted as nucleating agents for PP crystallization. The composites showed greater thermal degradation resistance as compared to PP. The composites showed a balance of stiffness and mechanical strength with increase of the flexural module and decrease of the tensile strength. The notched impact strength was not altered and the heat distortion temperature (HDT) of the composites was increased as compared to PP. The performance of the composites developed here showed that the industrial waste based on phenolic resin can be used as functional filler for PP. Furthermore, these composites are an alternative for the recycling of the phenolic resin industrial waste.

Keywords: Polypropylene composites, phenolic resin, recycling.

\section{Introdução}

O polipropileno (PP) isotático é um polímero termoplástico de ampla utilização para os mais diversos segmentos da indústria, desde embalagens até peças técnicas. O PP apresenta um conjunto de propriedades vantajosas com relação aos demais termoplásticos, tais como: equilíbrio de rigidez, resistência mecânica e tenacidade, resistência química, processabilidade, entre outras. É também um plástico reciclável e abundante para o reaproveitamento. O PP pode ser processado por diversos métodos tais como extrusão, injeção, sopro, rotomoldagem, compactação e termoformagem. A grande versatilidade de uso e a possibilidade de incorporação de cargas permitem que o PP seja cada vez mais empregado na forma de compósitos para os mais diversos fins ${ }^{[1]}$.

A resina fenólica é um polímero termorrígido sintetizado a partir da reação de policondensação entre fenol e solução de formaldeído, conhecido comumente como baquelite. A resina fenólica é utilizada sob a forma de compósitos com diversos tipos de cargas vegetais e minerais. Apresenta uma combinação de propriedades tais como boa estabilidade dimensional, elevada dureza, rigidez, resistência ao desgaste, ao calor e a agentes químicos. É um material que permite a fabricação de artefatos para diversas finalidades, tais como engrenagens, pastilhas de freio, cabos de panela, compensado naval e laminados de madeira ${ }^{[2-4]}$.

Basicamente, existem três tipos de processos de fabricação de artefatos com composições a base de resina fenólica, são eles: compactação, injeção e moldagem por transferência ${ }^{[5]}$. Em todos estes processos de fabricação há geração de resíduos de difícil reutilização, uma vez que este material é insolúvel e infusível, como resultado do processo de cura que ocorre durante o processamento. No Brasil, toda peça com defeito de moldagem, os canais de alimentação e as rebarbas oriundas do processo de transformação da resina não são utilizados, produzindo um conteúdo significativo de material sem destino nobre, permanecendo, quando coletado, como lixo, em aterros controlados. No exterior, a reciclagem da resina fenólica é ainda incipiente, sendo que a pirólise é o principal processo $^{[6-7]}$.

O desenvolvimento de compósitos com matriz termoplástica de PP carregada com resíduos industriais de resina fenólica criaria uma alternativa para o reaproveitamento desses resíduos e possibilitaria a produção de novos materiais compósitos, visando melhoraria das propriedades do $\mathrm{PP}^{[8]}$. Nesse contexto, Groning e colaboradores prepararam compósitos com matriz 
de polipropileno (PP) contendo resíduo de resina fenólica com fibra de vidro e estudaram os efeitos da incorporação do resíduo sobre a estabilização termo-oxidativa ${ }^{[9]}$ e o reforço mecânico ${ }^{[10]}$ dos materiais. Cui e colaboradores prepararam compósitos de PP contendo resina fenólica Novolac e negro de fumo e estudaram as propriedades elétricas dos materiais ${ }^{[11]}$.

O objetivo deste estudo foi analisar a viabilidade técnica do uso de resíduo industrial composto de resina fenólica, carga lignocelulósica, carbonato de cálcio, talco, mica e negro de fumo como carga para o polipropileno (PP). Compósitos de PP e resíduo de resina fenólica foram processados em extrusora de rosca dupla e moldados por injeção na forma de corpos de prova, os quais foram caracterizados quanto à microestrutura (MEV), as propriedades térmicas (DSC e TGA), mecânicas (tração, flexão e impacto) e temperatura de distorção ao calor (HDT).

\section{Experimental}

\section{Materiais}

O polipropileno (PP) é um homopolímero isotático comercial com código SM 6100, com densidade específica de 0,905 g. $\mathrm{cm}^{-3}$, fornecido pela Suzano Petroquímica.

O resíduo a base de resina fenólica e cargas foi obtido de uma indústria de fabricação de cabos de panela. São rejeitos na forma de rebarbas oriundas do processo de moldagem e cura por compressão em prensa hidráulica. As rebarbas possuíam formato de escamas irregulares, com dimensões aproximadas de $5 \mathrm{~cm}$ de largura e $1 \mathrm{~mm}$ de espessura. Segundo o fabricante, esse processo gera aproximadamente $10 \%$ de rejeitos. O custo para o descarte do resíduo é de $\mathrm{R} \$ 224,00$ (duzentos e vinte e quatro reais) para cada $5 \mathrm{~m}^{3}$ (cinco metros cúbicos) ou sete containers do resíduo.

\section{Moagem e caracterização do resíduo industrial de resina fenólica}

Os resíduos a base de resina fenólica e cargas foram triturados em moinho de bolas Retsch S100. Os tamanhos das partículas moídas do resíduo foram determinados por classificação granulométrica via peneiramento de acordo com a norma ASTM D1921 utilizando-se um peneirador automático Produtest modelo T, contendo 4 peneiras seqüenciais com aberturas de 20, 40, 70 e 100 mesh.

A densidade das partículas do resíduo moído foi determinada utilizando um picnômetro seguindo a norma ISO 8962.

O tipo e o percentual de cargas presentes no resíduo foram determinados por análise termogravimétrica (TGA) de acordo com a norma ASTM E1131 em equipamento TA Instruments Q500, na faixa de temperaturas entre $10^{\circ} \mathrm{C}$ e $700{ }^{\circ} \mathrm{C}$, a taxa de aquecimento de $10{ }^{\circ} \mathrm{C} / \mathrm{min}$ e atmosfera de $\mathrm{N}_{2}$ sob fluxo de $60 \mathrm{~mL} / \mathrm{min}$. Amostra ( 10 mg) foi analisada na forma de pó moído.

A composição química das cargas minerais presentes no resíduo de resina fenólica foi obtida por análise em microscópio eletrônico de varredura (MEV) Philips modelo XL30 FEG com espectrômetro de energia dispersiva (EDS).

\section{Processamento dos compósitos}

Os compósitos de PP carregados com resíduo de resina fenólica e cargas foram preparados em extrusora de rosca dupla co-rotacional inter-penetrante Teck-Tril modelo DCT 20, com D $=20 \mathrm{~mm}$ e $\mathrm{L} / \mathrm{D}=36$. Os compósitos preparados são descritos na Tabela 1. As condições de operação da extrusora de rosca dupla são apresentadas na Tabela 2.

Corpos de prova para os ensaios mecânicos de resistência à tração, resistência à flexão, resistência ao impacto Izod e temperatura de distorção ao calor (HDT) foram moldados a partir dos grânulos extrusados utilizando uma máquina injetora ARBURG modelo Allrounder 270S 400-170, com moldes padronizados de acordo com as dimensões estabelecidas nas respectivas normas ASTM D638, D790, D256 e D648. As condições operacionais da moldagem por injeção dos compósitos são apresentadas na Tabela 3. Para cada formulação moldada, registrou-se a pressão de preenchimento necessária para a realização da comutação para a etapa de recalque, nas condições de injeção empregadas.

\section{Caracterização dos compósitos}

Os valores de índice de fluidez (MFI) dos grânulos extrusados foram determinados utilizando-se o equipamento Dynisco Kayeness Polymer Test Systems modelo LMI 4003, a temperatura do barril de $230{ }^{\circ} \mathrm{C}$, sob carga de $2,16 \mathrm{~kg}$, segundo a norma ASTM D1238.

As microestruturas dos compósitos foram investigadas em microscópio eletrônico de varredura (MEV) Philips modelo XL30 FEG, operado com voltagem de aceleração de $25 \mathrm{kV}$. As amostras para estas análises foram obtidas a partir da superfície de fratura criogênica de corpos de prova de impacto, que foram recobertas por uma fina camada de ouro.

As temperaturas de fusão e cristalização do polipropileno (PP) nos compósitos foram analisadas por calorimetria diferencial de varredura (DSC) de acordo com a norma ASTM D3418 em equipamento TA Instruments modelo Q100, na faixa de temperaturas entre $50{ }^{\circ} \mathrm{C}$ e $200{ }^{\circ} \mathrm{C}$, com programa aquecimento-resfriamento, a uma taxa de $10{ }^{\circ} \mathrm{C} / \mathrm{min}$, sob atmosfera de $\mathrm{N}_{2}$ com fluxo de $50 \mathrm{~mL} /$ min. Amostras $(\sim 5 \mathrm{mg})$ foram retiradas do centro geométrico de corpos de prova de impacto moldados por injeção.

$\mathrm{O}$ grau de cristalinidade percentual $\left(\mathrm{X}_{\mathrm{c}}\right)$ do PP nos materiais foi calculado a partir da equação 1 :

Tabela 1. Compósitos de polipropileno e resina fenólica estudados.

\begin{tabular}{cc}
\hline Designação & Descrição \\
\hline PP & Polipropileno puro \\
PP10 & $\begin{array}{c}\text { Compósito de polipropileno com 10\% em massa de } \\
\text { resíduo industrial de resina fenólica com cargas }\end{array}$ \\
PP15 & $\begin{array}{c}\text { Compósito de polipropileno com 15\% em massa de } \\
\text { resíduo industrial de resina fenólica com cargas } \\
\text { PP20 } \\
\text { Compósito de polipropileno com 20\% em massa de } \\
\text { resíduo industrial de resina fenólica com cargas }\end{array}$ \\
\hline
\end{tabular}

Tabela 2. Condições de preparação dos compósitos em extrusora de rosca dupla.

\begin{tabular}{lc}
\hline \multicolumn{1}{c}{ Parâmetro } & Valor \\
\hline Temperatura do barril & $200{ }^{\circ} \mathrm{C}$ \\
Rotação das roscas & $300 \mathrm{rpm}$ \\
Taxa de alimentação & $7 \mathrm{~kg} / \mathrm{h}$ \\
\hline
\end{tabular}

Tabela 3. Condições de moldagem dos compósitos por injeção.

\begin{tabular}{lc}
\hline \multicolumn{1}{c}{ Parâmetro } & Valor \\
\hline Perfil de temperaturas do barril & $210-220-230-230{ }^{\circ} \mathrm{C}$ \\
Vazão de injeção & $50 \mathrm{~cm}^{3} / \mathrm{s}$ \\
Comutação para recalque & $95 \mathrm{vol} . \%$ \\
Pressão de recalque & $300 \mathrm{bar}$ \\
Tempo de recalque & $7 \mathrm{segundos}$ \\
Temperatura do molde & $50{ }^{\circ} \mathrm{C}$ \\
Tempo de resfriamento & $30 \mathrm{segundos}$ \\
Força de fechamento & $250 \mathrm{kN}$ \\
\hline
\end{tabular}




$$
X_{C}=\frac{\Delta H_{m}}{\Delta H_{100 \%} \times \varphi} \times 100 \%
$$

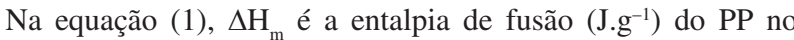
material determinada por DSC; $\Delta \mathrm{H}_{100 \%}$ é a entalpia de fusão do PP hipoteticamente $100 \%$ cristalino, cujo valor utilizado foi de 209 J.g ${ }^{-1[12]} ; \phi$ é a fração em massa do PP no compósito.

As temperaturas de decomposição térmica dos compósitos foram investigadas por análise termogravimétrica (TGA) de acordo com a norma ASTM E1131 em equipamento TA Instruments modelo Q100, na faixa de temperaturas entre $25{ }^{\circ} \mathrm{C}$ e $700{ }^{\circ} \mathrm{C}$, a uma taxa de aquecimento de $10^{\circ} \mathrm{C} / \mathrm{min}$, sob atmosfera de $\mathrm{N}_{2}$ com fluxo de $50 \mathrm{~mL} / \mathrm{min}$. Amostras $(\sim 10 \mathrm{mg})$ foram retiradas do centro geométrico de corpos de prova de impacto moldados por injeção.

Os compósitos moldados foram submetidos a ensaios mecânicos. Os corpos de prova foram acondicionados por 48 horas em uma sala com temperatura $\left(23^{\circ} \mathrm{C} \pm 2{ }^{\circ} \mathrm{C}\right)$ e umidade relativa $(50 \% \pm 5 \%)$ controladas, anteriormente a cada ensaio.

Os ensaios de resistência à tração foram realizados segundo a norma ASTM D638 em corpos de prova com geometria tipo I. Utilizou-se uma máquina de ensaios universal EMIC modelo DL 3000 , operada a uma velocidade de travessa de $5 \mathrm{~mm} / \mathrm{min}$. Os ensaios de resistência à flexão foram realizados segundo a norma ASTM D790. Utilizou-se uma máquina de ensaios universal EMIC modelo DL 3000, operada a uma velocidade de travessa de $1 \mathrm{~mm} / \mathrm{min}$. Os ensaios de resistência ao impacto Izod sob entalhe foram realizados de acordo com a norma ASTM D 256. Utilizou-se pêndulo CEAST modelo Resil Impactor com energia máxima de 2 J. Nesses ensaios, foram utilizados cinco corpos de prova para cada formulação, assumindo-se como resultado o valor médio obtido para cada teste, juntamente com o desvio padrão das medidas.

Os ensaios para determinação da temperatura de distorção ao calor (HDT) dos compósitos moldados foram realizados de acordo com a norma ASTM D648 em equipamento Tinius Olsen. Utilizou-se uma taxa de aquecimento de $2{ }^{\circ} \mathrm{C} / \mathrm{min}$ e uma carga de 1,80 MPa. Para cada composição ensaiada, foram testados três corpos de prova, assumindo-se como resultado o valor médio obtido para cada análise, juntamente com o desvio padrão das medidas.

\section{Resultados e Discussões}

\section{Caracterização do resíduo industrial de resina fenólica}

As frações em massa retidas de cada faixa de tamanhos das partículas moídas do resíduo industrial de resina fenólica, determinadas por classificação granulométrica, são mostradas na Tabela 4.

O resíduo moído apresentou densidade específica de $1,52 \pm 0,01 \mathrm{~g} \cdot \mathrm{cm}^{-3}$.

A composição quantitativa do resíduo industrial de resina fenólica foi determinada por TGA com base nas faixas de temperatura de decomposição características de cada componente presente no resíduo. Os resultados são apresentados na Tabela 5 .

A Figura 1a apresenta uma micrografia de MEV com ampliação de 200 vezes, obtida com detector de elétrons secundários (SE) de uma partícula do resíduo industrial de resina fenólica. Essa imagem apresenta detalhes do formato e do tamanho da partícula do resíduo. A Figura 1b é uma imagem ampliada (500 vezes) dessa partícula, obtida com detector de elétrons retro-espalhados (BSE). Observa-se nessa imagem a presença de cargas inorgânicas (regiões contrastadas em claro) dispersas na matriz de resina fenólica. A Figura 1c apresenta o espectro de EDS e a composição química das cargas presentes na partícula do resíduo. Baseado na presença desses elementos químicos, nos dados de composição obtidos no TGA e na experiência dos autores sugere-se a presença das seguintes cargas na composição do resíduo de resina fenólica: carbonato de cálcio $\left(\mathrm{CaCO}_{3}\right)$, talco $\left(\mathrm{Mg}_{3} \mathrm{Si}_{4} \mathrm{O}_{10}(\mathrm{OH})_{2}\right)$, mica $\left(\mathrm{K}_{2}\left(\mathrm{Al}_{2} \mathrm{O}_{3}\right)_{3}\left(\mathrm{SiO}_{2}\right) \cdot 6 \mathrm{H}_{2} \mathrm{O}\right) \mathrm{e}$ lignocelulose. Análise visual revelou ainda a presença de negro de fumo como pigmento preto.

\section{Caracterização dos compósitos de PP e resina fenólica}

Os valores de índice de fluidez (MFI) do PP e dos compósitos extrusados são apresentados na Tabela 6. Considerando-se os valores médios e os desvios padrão das medidas é possível observar diminuição $(\sim 10 \%)$ dos valores de fluidez dos compósitos, com relação ao PP puro.

A Tabela 7 apresenta as pressões de comutação necessárias para a moldagem dos compósitos por injeção. No processamento por injeção, observou-se aumento de até $10 \%$ dos valores de pressão de comutação para os compósitos contendo teores mais elevados de resíduo de resina fenólica, sugerindo assim que houve aumento pequeno da viscosidade dos compósitos com o aumento do teor de resíduo incorporado. Estes resultados corroboram os valores de índice de fluidez (MFI) dos materiais extrusados (Tabela 6). A pequena alteração na viscosidade dos compósitos com relação ao PP puro é interessante do ponto de vista de vislumbrar a moldagem de peças técnicas a partir dos compósitos desenvolvidos nesse estudo.

A Figura 2 apresenta as micrografias obtidas em MEV com detector de elétrons retro-espalhados (ampliação de 50 vezes) das superfícies de fratura dos compósitos moldados. As micrografias de MEV revelam que as partículas do resíduo apresentam geometrias não uniformes e tamanhos inferiores a $500 \mu \mathrm{m}$. Comparando-se o tamanho dessas partículas nos compósitos com o tamanho inicial das partículas do resíduo moído (Tabela 4), conclui-se que o processamento adotado para os compósitos em máquina extrusora e injetora não foi capaz de produzir dispersão significativa do resíduo de resina fenólica com carga na matriz de PP. Esse desempenho é explicado em decorrência do caráter termorrígido (infusível) da resina fenólica no resíduo, o que dificulta sua dispersão na matriz de PP. No caso, a pequena redução do tamanho das partículas do resíduo nos compósitos deve-se à quebra mecânica oriunda de processos deformacionais ocorridos ao longo da rosca da extrusora e injetora.

Tabela 4. Frações em massa retidas de cada faixa de tamanhos das partículas moídas do resíduo industrial de resina fenólica determinadas por classificação granulométrica.

\begin{tabular}{cc}
\hline $\begin{array}{c}\text { Faixa de tamanho de } \\
\text { partícula }(\boldsymbol{\mu m})\end{array}$ & $\begin{array}{c}\text { Percentual retido } \\
\text { (em massa) }\end{array}$ \\
\hline$<150$ & $4,7 \%$ \\
$150-212$ & $13 \%$ \\
$212-420$ & $24,5 \%$ \\
$420-850$ & $35,4 \%$ \\
$>850$ & $22,4 \%$ \\
\hline
\end{tabular}

Tabela 5. Composição do resíduo industrial de resina fenólica determinada por TGA.

\begin{tabular}{ccccc}
\hline Componente & $\begin{array}{c}\text { Resina } \\
\text { fenólica } \\
\text { Novolac }\end{array}$ & $\begin{array}{c}\text { Carga } \\
\text { lignocelulósica }\end{array}$ & $\begin{array}{c}\text { Carga } \\
\text { inorgânica }\end{array}$ & Voláteis \\
\hline $\begin{array}{l}\text { Percentual } \\
\text { (em massa) }\end{array}$ & $22 \%$ & $19 \%$ & $52 \%$ & $7 \%$ \\
\hline
\end{tabular}


A Figura 3 apresenta as curvas de fusão (3a) e de cristalização (3b) obtidas por DSC para os compósitos moldados. A Tabela 8 apresenta as temperaturas de pico de fusão $\left(\mathrm{T}_{\mathrm{m}}\right)$ e de pico de cristalização $\left(T_{c}\right)$ e o grau de cristalinidade $\left(X_{c}\right)$ do PP nos materiais. Observa-se que a temperatura de pico de fusão $\left(\mathrm{T}_{\mathrm{m}}\right)$ do PP não foi alterada em decorrência da incorporação do resíduo de resina fenólica com cargas. No entanto, as entalpias de fusão, normalizadas com relação à fração de PP nos materiais, são maiores nos compósitos, caracterizando aumento do grau de cristalinidade (X) do PP nos compósitos, com relação ao PP puro. A presença do resíduo de resina fenólica com carga causou aumento na temperatura de pico de cristalização $\left(\mathrm{T}_{\mathrm{c}}\right)$ do $\mathrm{PP}$ nos compósitos.

Os resultados de DSC (Figura 3 e Tabela 8) sugerem que as partículas de resina fenólica contendo cargas atuaram como agentes de nucleação heterogênea do PP, acelerando sua cristalização e conduzindo a maiores valores de grau de cristalinidade nos compósitos moldados. Efeito de nucleação da cristalização do PP em compósitos contendo cargas inorgânicas tais como carbonato de cálcio e talco, as quais estão presentes no resíduo de resina fenólica empregado nesse estudo, é bastante comum de ocorrer ${ }^{[13]}$.

A Figura 4 apresenta as curvas de TGA dos compósitos moldados. Na Figura 4a são apresentadas as curvas de perda de massa e na Figura 4b são apresentadas as curvas de derivada da perda

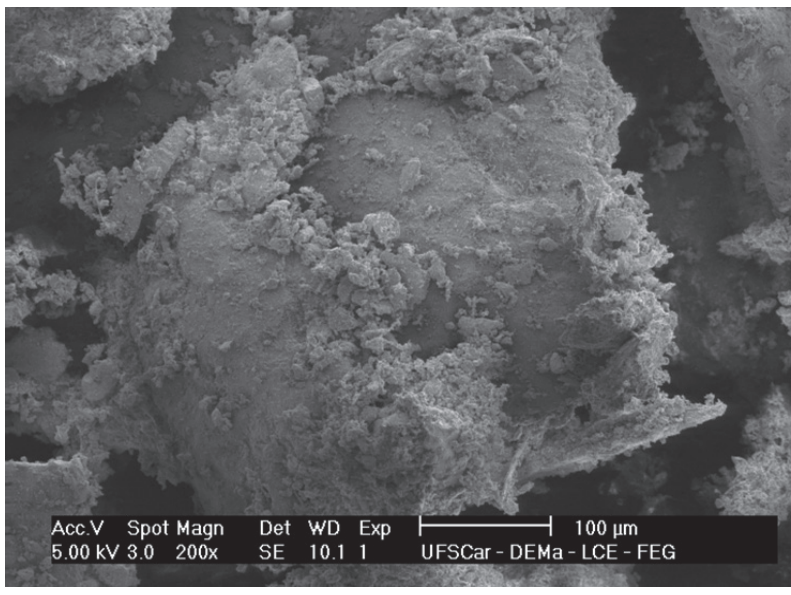

(a)
Tabela 6. Índice de fluidez (MFI) dos materiais extrusados.

\begin{tabular}{ccccc}
\hline Amostra & PP & PP10 & PP15 & PP20 \\
\hline MFI (g/10 min $)$ & $16,5 \pm 0,8$ & $14,9 \pm 0,7$ & $14,5 \pm 0,9$ & $14,2 \pm 0,9$ \\
\hline
\end{tabular}

Tabela 7. Valores de pressão de comutação para moldagem por injeção dos compósitos

\begin{tabular}{cc}
\hline Material & Pressão de comutação \\
\hline PP & 660 bar \\
PP10 & $690 \mathrm{bar}$ \\
PP15 & 705 bar \\
PP20 & $730 \mathrm{bar}$ \\
\hline
\end{tabular}

Tabela 8. Temperaturas de pico de fusão $\left(\mathrm{T}_{\mathrm{m}}\right)$, de pico de cristalização $\left(\mathrm{T}_{\mathrm{c}}\right) \mathrm{e}$ grau de cristalinidade $\left(\mathrm{X}_{\mathrm{c}}\right)$ do PP nos compósitos.

\begin{tabular}{cccc}
\hline Amostra & $\mathbf{T}_{\mathrm{m}}\left({ }^{\circ} \mathbf{C}\right)$ & $\mathbf{T}_{\mathbf{c}}\left({ }^{\circ} \mathbf{C}\right)$ & $\mathbf{X}_{\mathbf{c}}(\%)$ \\
\hline PP & 162,6 & 111,7 & 30,5 \\
PP10 & 162,2 & 114,7 & 31,7 \\
PP15 & 162,0 & 115,6 & 39,2 \\
PP20 & 162,4 & 117,5 & 39,7 \\
\hline
\end{tabular}

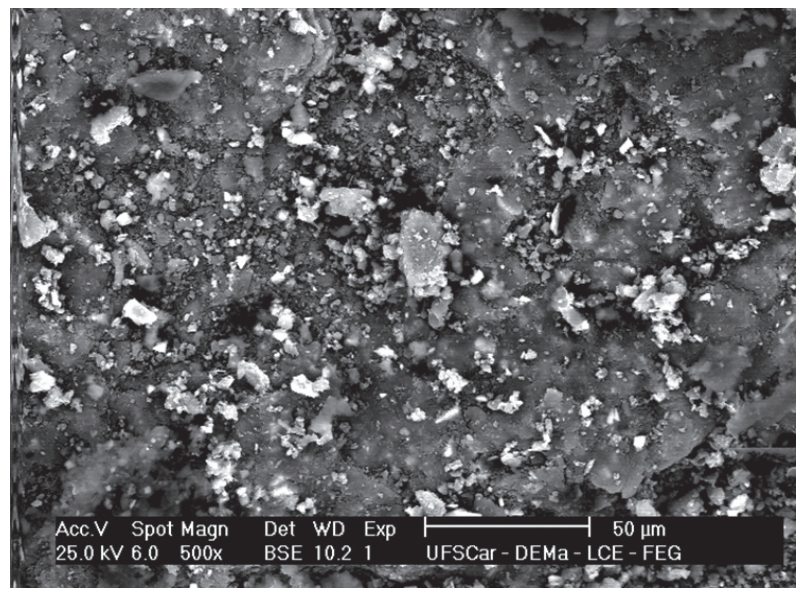

(b)

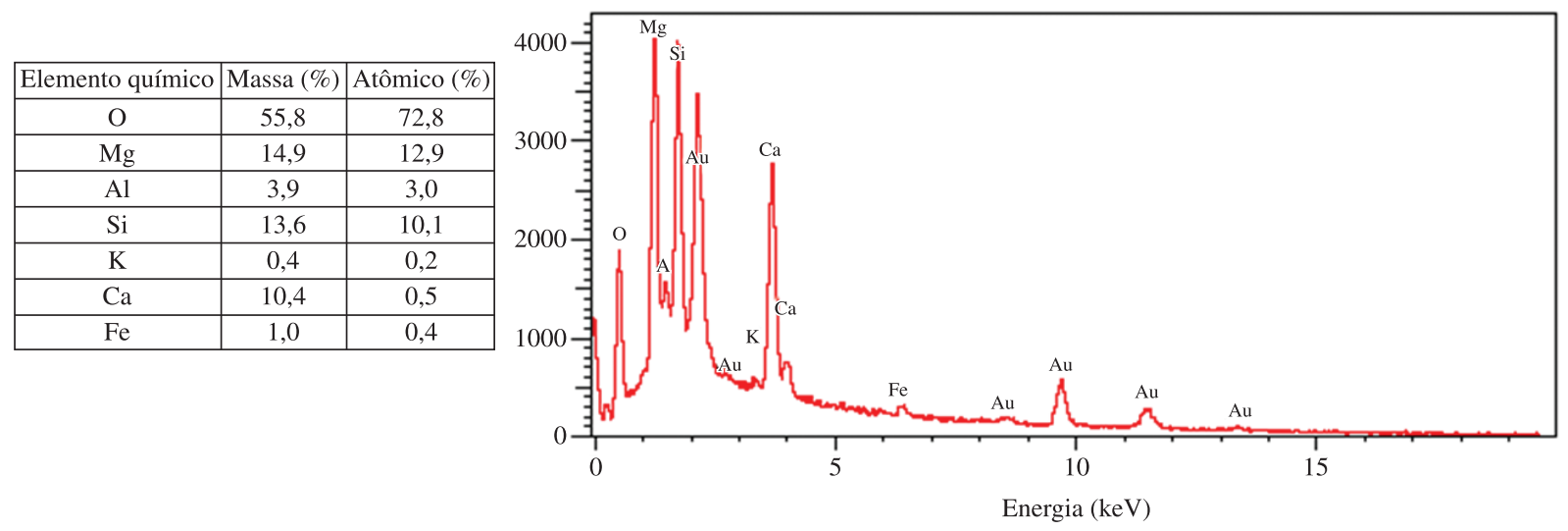

(c)

Figura 1. Micrografias obtidas em MEV/EDS de partícula do resíduo industrial de resina fenólica com cargas: (a) detalhe do formato e tamanho da partícula (ampliação de 200x); (b) detalhe das cargas presentes na partícula (ampliação de 500x); (c) espectro de EDS e composição química das cargas 


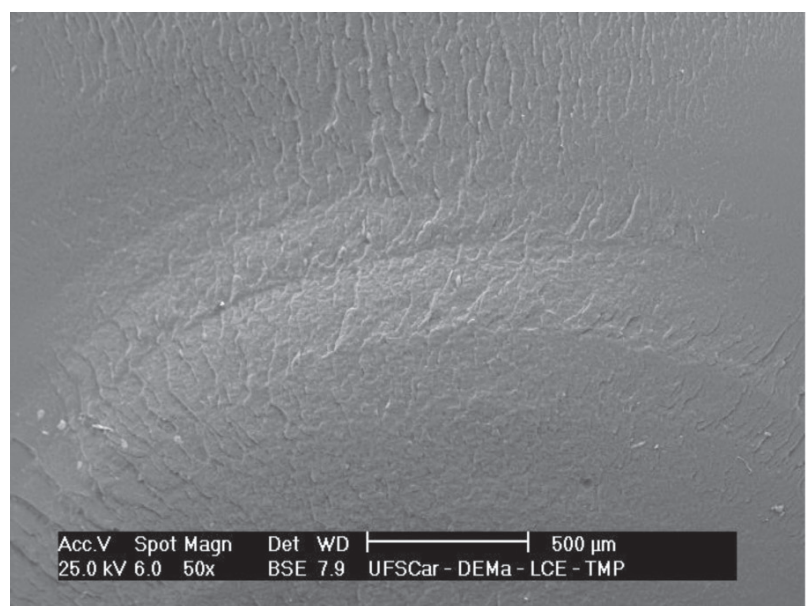

(a) PP

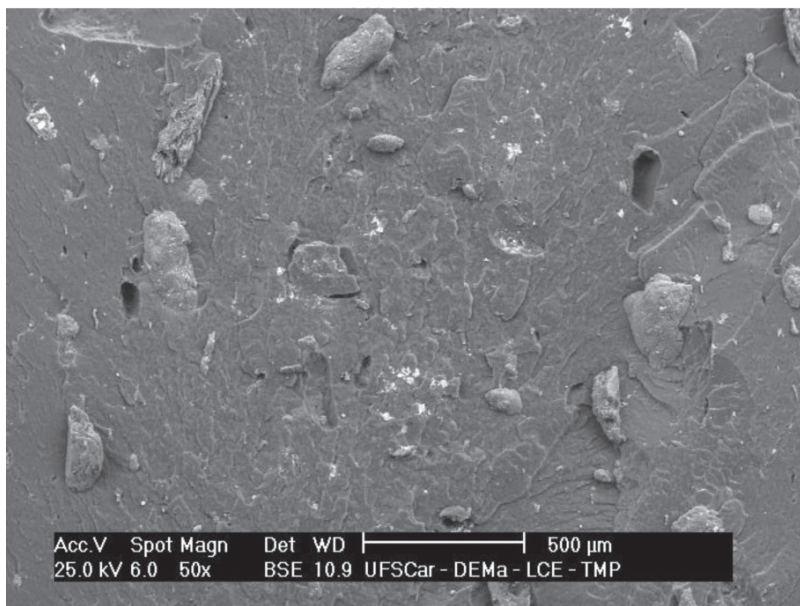

(c) PP15

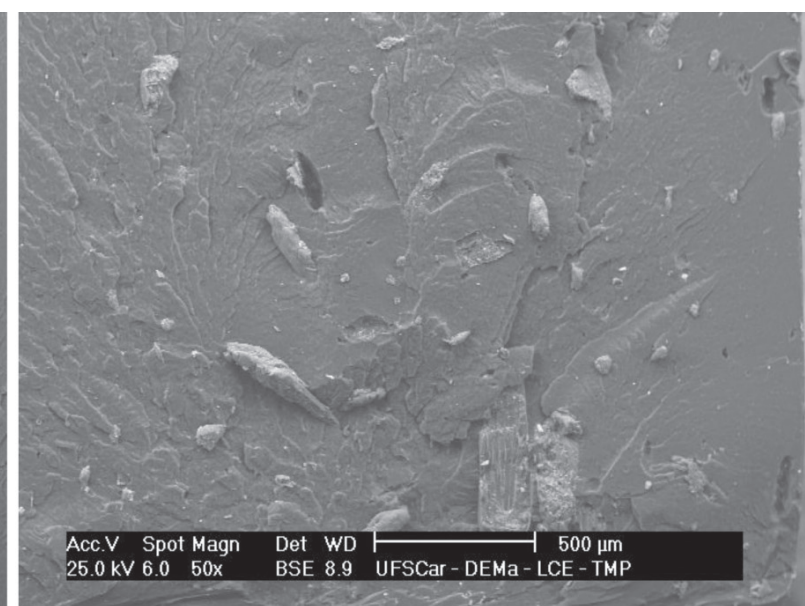

(b) PP10

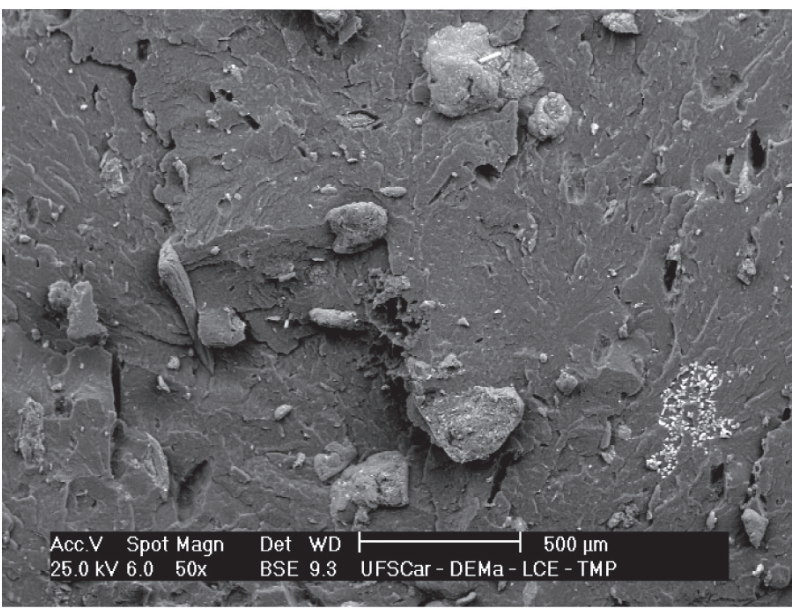

(d) PP20

Figura 2. Micrografias obtidas em MEV de superfícies de fratura dos compósitos moldados.

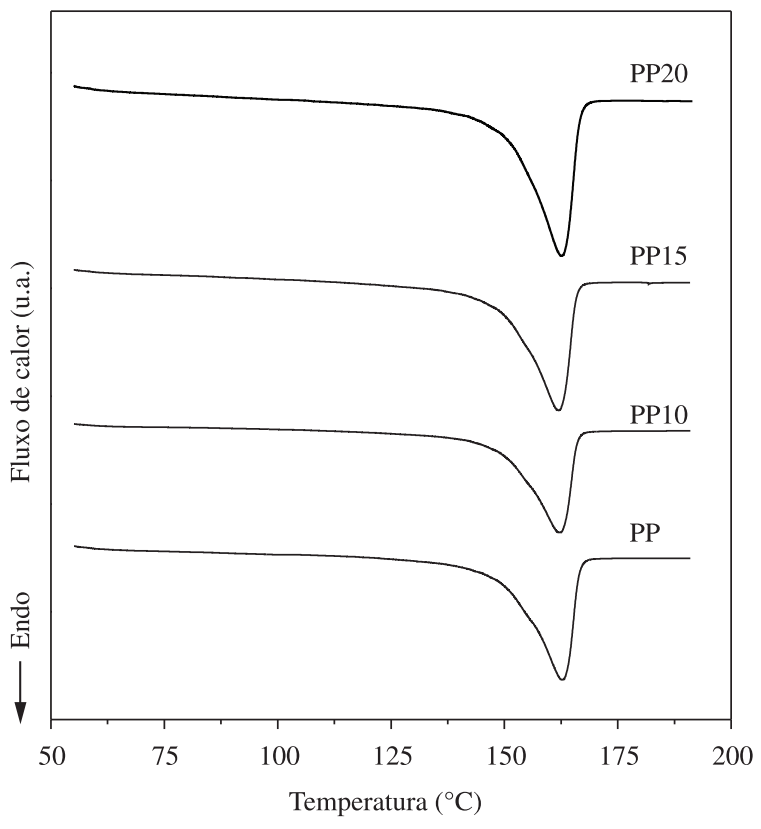

(a)

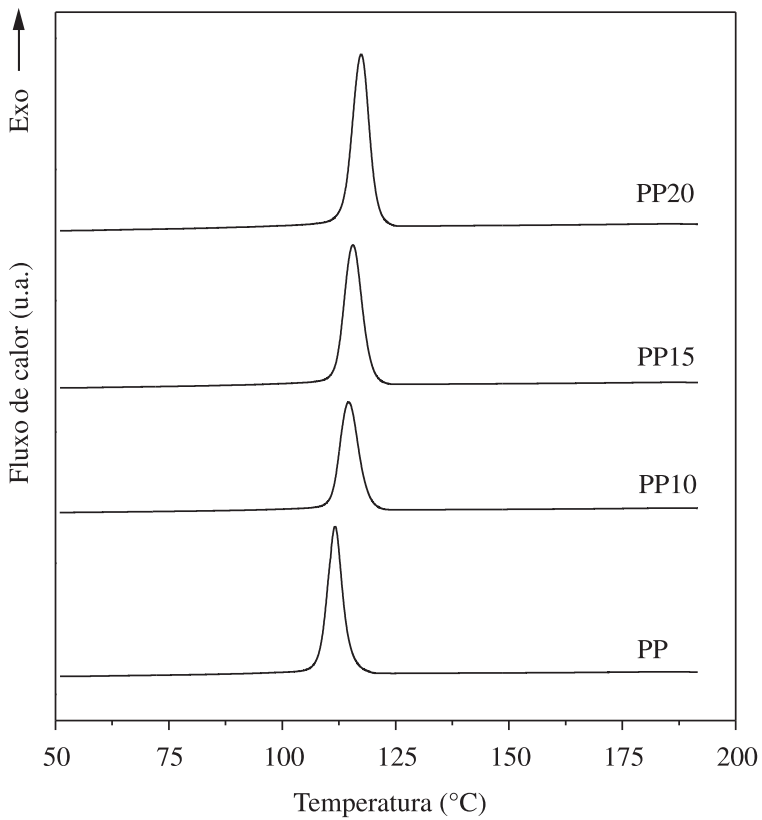

(b)

Figura 3. Curvas de DSC dos compósitos: a) fusão; b) cristalização. 


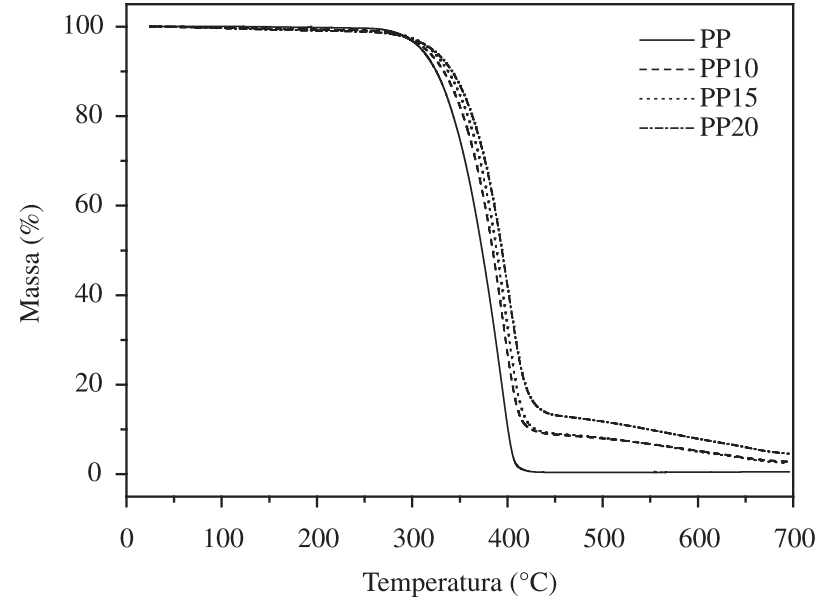

(a)

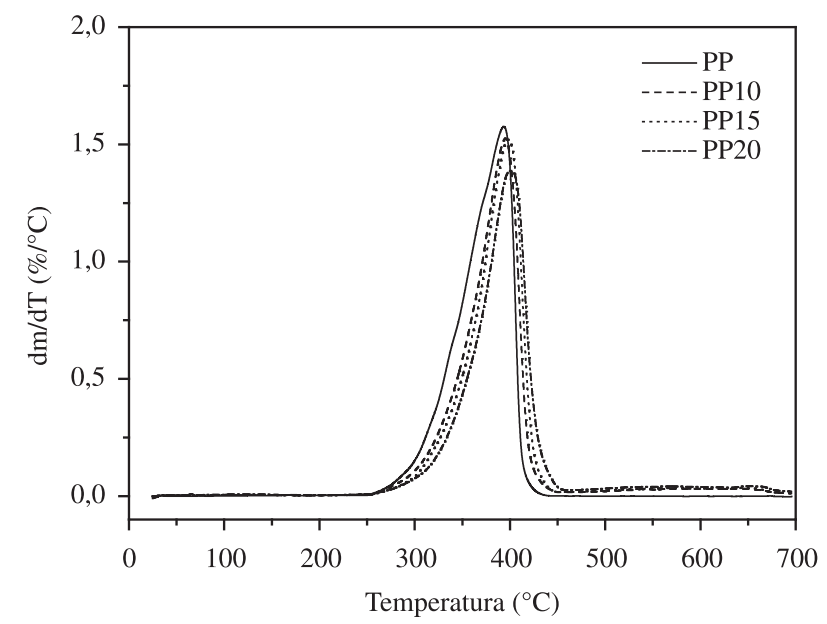

(b)

Figura 4. Curvas de TGA dos compósitos: a) perda de massa; b) derivada da perda de massa com relação à temperatura.

de massa com relação à temperatura. Na Tabela 9 são apresentadas as temperaturas de início $\left(\mathrm{T}_{\text {ińcio }}\right)$, de taxa máxima $\left(\mathrm{T}_{\text {máx }}\right)$ e do final $\left(\mathrm{T}_{\text {final }}\right)$ do processo degradativo do PP nos materiais.

Observou-se que a incorporação de resíduo de resina fenólica com cargas contribuiu para aumentar a temperatura máxima de degradação do PP nos compósitos, bem como para que as temperaturas de início e término de degradação seguissem a mesma tendência, alcançando valores maiores para os compósitos contendo teores maiores de resíduo de resina fenólica com cargas. Groning e colaboradores ${ }^{[9]}$ observaram que a incorporação de resíduo de resina fenólica contendo fibra de vidro em compósitos com matrizes de PP causa efeito antioxidante nos materiais, e atribuíram à esse efeito a estrutura de fenol impedido da resina fenólica.

Os valores do módulo sob flexão dos compósitos são apresentados na Figura 5. Observa-se aumento desta propriedade com a incorporação de quantidades crescentes de resíduo de resina fenólica com cargas nos compósitos, conferindo assim um aumento da rigidez ao PP. Esse aumento é explicado devido às características de composição do resíduo, que contém resina fenólica, celulose e cargas inorgânicas, todos com rigidez superior ao PP. Outro aspecto importante que contribui para o aumento da rigidez do PP nos compósitos é o fato do grau de cristalinidade do PP ter sido aumentado com a incorporação do resíduo, como observado nos ensaios de DSC (Figura 3 e Tabela 8).

Talco e carbonato de cálcio, presentes no resíduo, são cargas comumente empregadas em compósitos com PP por apresentarem processabilidade e acrescentarem propriedades ao PP como rigidez mecânica e resistência termo-mecânica, além de serem abundantes e baratas ${ }^{[14]}$. O incremento do valor do módulo de flexão do compósito PP20 desse estudo é cerca da metade dos valores obtidos na literatura para compósitos de $\mathrm{PP}$ com $20 \%$ de $\mathrm{CaCO}_{3}$ (tamanho de partícula de $\sim 25 \mu \mathrm{m})^{[15]}$ e para compósitos de PP com $20 \%$ de talco (tamanho de partícula de $\sim 10 \mu \mathrm{m})^{[16]}$. Esse comparativo sugere que há um potencial de melhoria da rigidez dos compósitos em estudo a ser explorado via refinamento da morfologia de fases. Esse refinamento pode ser atingido via micronização do resíduo de resina fenólica com cargas a partículas com tamanhos inferiores aos obtidos nesse estudo, em etapa anterior à da extrusão dos materiais.

A Figura 6 apresenta os valores de resistência à tração dos compósitos. Observa-se um decréscimo desta propriedade com a incorporação do resíduo de resina fenólica aos compósitos. Este comportamento é explicado devido ao fato de que o PP apresentou escoamento nítido nos ensaios de tração enquanto que

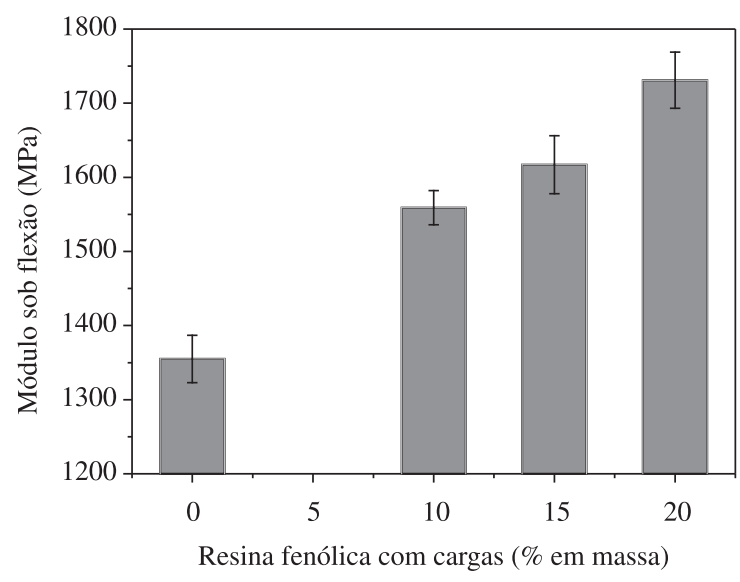

Figura 5. Módulo sob flexão dos compósitos.

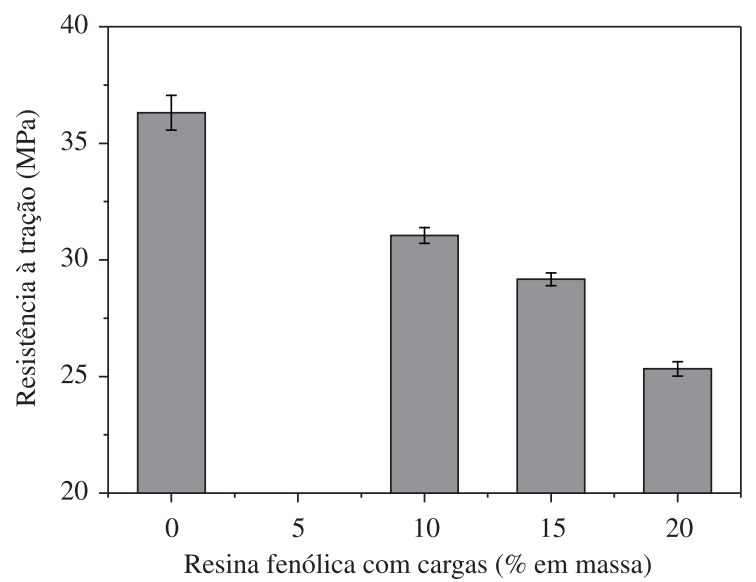

Figura 6. Resistência à tração dos compósitos.

Tabela 9. Temperaturas de degradação do PP nos compósitos (TGA).

\begin{tabular}{cccc}
\hline Amostra & $\mathbf{T}_{\text {início }}\left({ }^{\circ} \mathbf{C}\right)$ & $\mathbf{T}_{\text {máx }}\left({ }^{\circ} \mathbf{C}\right)$ & $\mathbf{T}_{\text {final }}\left({ }^{\circ} \mathbf{C}\right)$ \\
\hline PP & 257 & 393 & 440 \\
PP10 & 261 & 395 & 449 \\
PP15 & 263 & 398 & 453 \\
PP20 & 265 & 401 & 455 \\
\hline
\end{tabular}




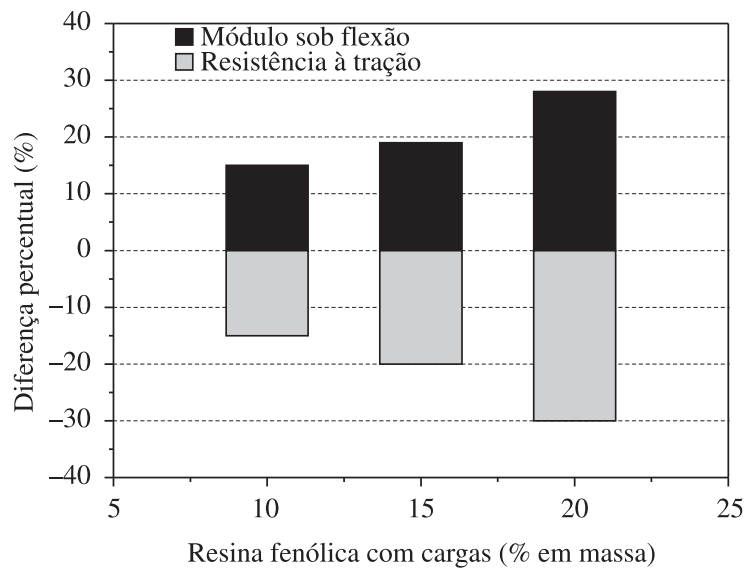

Figura 7. Variação dos valores de módulo de flexão e de resistência à tração dos compósitos.

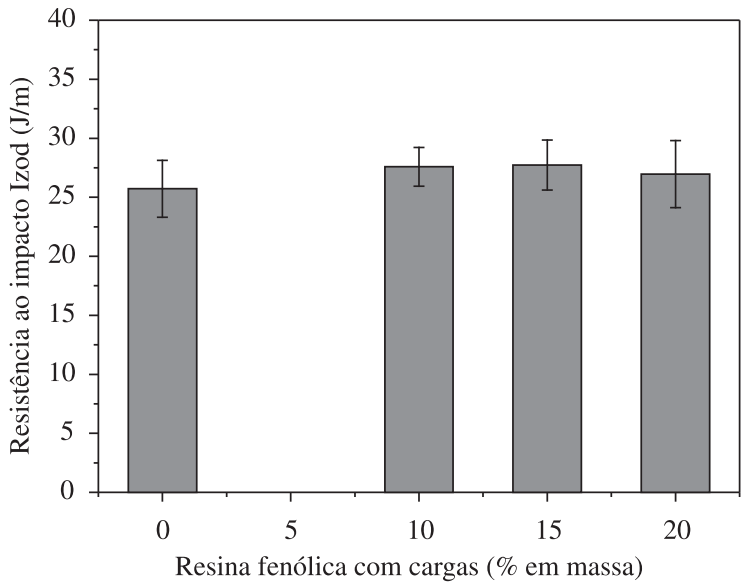

Figura 8. Resistência ao impacto Izod sob entalhe dos compósitos.

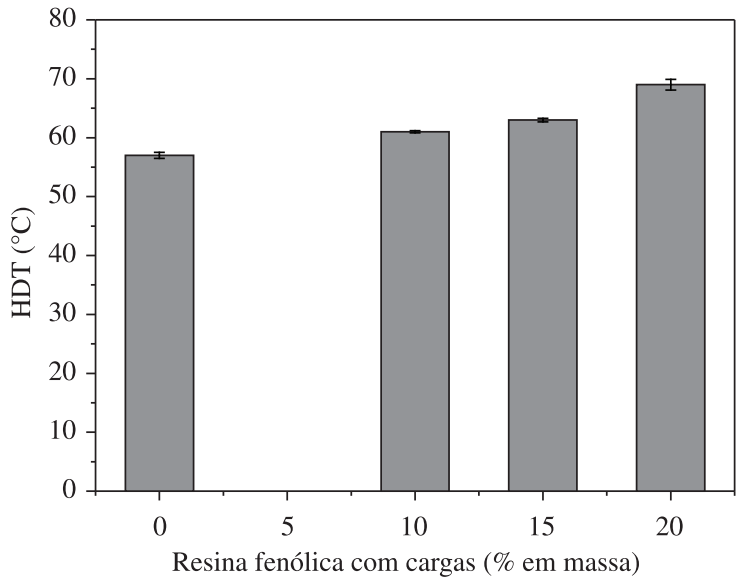

Figura 9. Temperatura de distorção ao calor (HDT) dos compósitos.

os compósitos romperam prematuramente, ou seja, a incorporação do resíduo inibiu o escoamento plástico em larga escala do PP. Esse fato ocorreu devido à dispersão insatisfatória do resíduo na matriz do PP, o que resultou em baixa adesão interfacial nos compósitos.

A Figura 7 mostra que o aumento percentual relativo do módulo de flexão é acompanhado pelo decréscimo da resistência à tração de magnitude semelhante, ou seja, a incorporação do resíduo de resina fenólica com cargas causou um balanço de rigidez e resistência à tração ao material compósito.
Os valores de resistência ao impacto Izod sob entalhe dos compósitos são apresentados na Figura 8. Observou-se que a incorporação do resíduo de resina fenólica contendo cargas não alterou esta propriedade com relação ao PP puro.

Os valores das temperaturas de distorção ao calor (HDT) dos compósitos são apresentados na Figura 9. Observou-se aumento da temperatura de distorção ao calor (HDT) dos compósitos com o aumento do percentual incorporado de resíduo de resina fenólica com cargas, o que é coerente com o aumento do módulo sob flexão dos compósitos (Figura 5).

\section{Conclusões}

Compósitos de matriz de polipropileno (PP) carregada com resíduo de resina fenólica com cargas lignocelulósicas e inorgânicas (carbonato de cálcio, talco e mica) foram obtidos por processamento em extrusora de rosca dupla seguido de moldagem por injeção. Esses compósitos foram caracterizados por microscopia eletrônica de varredura (MEV), análises térmicas (DSC e TGA), ensaios mecânicos (tração, flexão e impacto) e ensaios termo-mecânicos (HDT). Os compósitos moldados apresentaram partículas do resíduo com tamanhos inferiores a $500 \mu \mathrm{m}$ dispersas na matriz de PP. A incorporação do resíduo nos compósitos com PP produziu pequeno aumento $(\sim 10 \%)$ da viscosidade dos compósitos com relação ao PP, baseado na pressão de injeção. As partículas do resíduo atuaram como agentes de nucleação da cristalização do PP nos compósitos moldados. Os compósitos apresentaram maior resistência à degradação térmica com relação ao PP. Os compósitos apresentaram um balanço de rigidez e resistência com aumento do módulo sob flexão e diminuição da resistência à tração. A incorporação do resíduo não alterou a resistência ao impacto sob entalhe dos compósitos. Os compósitos apresentaram temperaturas de distorção ao calor (HDT) superiores ao PP. O desempenho dos compósitos desenvolvidos demonstrou que o resíduo industrial de resina fenólica atua como uma carga funcional para o PP. Além disso, os compósitos desenvolvidos representam uma alternativa de reciclagem para o resíduo industrial de resina fenólica.

\section{Referências Bibliográficas}

1. Moore, P. E. - "Polypropylene Handbook: Polymerization, characterization, properties, aplications", Hanser, Munich (1996).

2. Mano, E. B. - "Polímeros como materiais de engenharia", Edgard Blücher, São Paulo (2000).

3. Goodman, S. H. - "Handbook of Thermosetting Plastics", Noyes Publication, New Jersey (1998).

4. Domininghaus, H. - "Plastics for engineers", Hanser, Munich (1993).

5. Lubin, G. - "Handbook of Fiberglass and Advanced Plastics Composites", Van Nostrand Reinhold Company, New York (1969).

6. Pickering, S. J. - Compos. Part A, Appl. Sci. Manuf., 37, p.1206 (2006). http://dx.doi.org/10.1016/j.compositesa.2005.05.030

7. Cunliffe, A. M.; Jones, N. \& Williams, P. T. - J. Anal. Appl. Pyrolys., 70, p.315 (2003). http://dx.doi.org/10.1016/S0165-2370(02)00161-4

8. Cavalcante, A. P. - "Compósitos de polipropileno e resíduo industrial de resina fenólica”, Dissertação de Mestrado, Universidade Federal do Rio de Janeiro, Brasil (2010).

9. Groning, M.; Eriksson, H.; Hakkarainen, M. \& Albertsson, A. C. - Polym. Degrad. Stab., 91, p.1815 (2006).

10. Groning, M.; Hakkarainen, M. \& Albertsson, A. C. - Polym. Adv. Technol., 12, p. 501 (2004).

11. Cui, L.; Zhang, Y.; Zhang, Y. \& Zhang, X. - Eur. Polym. J., 43, p.5097 (2007). http://dx.doi.org/10.1016/j.eurpolymj.2007.08.023 
12. Silva, A. L. N.; Rocha, M. C. G.; Guimarães, M. J. O. C.; Lovisi, H.; Coutinho, F. M. B. \& Santa Maria, L. C. - Polímeros, 11, p.135 (2001). http://dx.doi.org/10.1590/S0104-14282001000300013

13. McGenity, P. M.; Hooper, J. J.; Paynter, C. D.; Riley, A. M.; Nutbeem, C.; Elton, N. J. \& Adams, J. M. - Polymer, 33, p.5215 (1992). http:// dx.doi.org/10.1016/0032-3861(92)90804-6
14. Jikan, S. S.; Samsudin, M. S. F.; Ariff, Z. M.; Ishak, Z. A. M. \& Ariffin, A. - J. Reinf. Plast. Compos., 28, p.2577 (2009). http://dx.doi. org/10.1177/0731684408092440

15. Yang, K.; Yang, Q.; Li, G.; Sun, Y. \& Feng, D. - Polym. Compos., 27, p.443 (2006). http://dx.doi.org/10.1002/pc.20211

16. Shelesh-Nezhad, K. \& Taghizadeh, A. - Polym. Eng. Sci., 47, p.2124 (2007). http://dx.doi.org/10.1002/pen.20940

Enviado: 17/01/11 Reenviado: $20 / 07 / 11$

Aceito: $26 / 08 / 11$ 